\title{
Breakdown of the local density approximation in interacting systems of cold fermions in strongly anisotropic traps
}

\section{Citation}

Imambekov, Adilet, C. J. Bolech, Mikhail Lukin, and Eugene Demler. 2006. Breakdown of the Local Density Approximation in Interacting Systems of Cold Fermions in Strongly Anisotropic Traps. Physical Review A 74, no. 5. doi:10.1103/physreva.74.053626.

\section{Published Version}

doi:10.1103/PhysRevA.74.053626

\section{Permanent link}

http://nrs.harvard.edu/urn-3:HUL.InstRepos:27896442

\section{Terms of Use}

This article was downloaded from Harvard University's DASH repository, and is made available under the terms and conditions applicable to Other Posted Material, as set forth at http:// nrs.harvard.edu/urn-3:HUL.InstRepos:dash.current.terms-of-use\#LAA

\section{Share Your Story}

The Harvard community has made this article openly available.

Please share how this access benefits you. Submit a story.

\section{Accessibility}




\title{
Breakdown of the local density approximation in interacting systems of cold fermions in strongly anisotropic traps
}

\author{
Adilet Imambekov, ${ }^{1}$ C. J. Bolech, ${ }^{1,2}$ Mikhail Lukin, ${ }^{1}$ and Eugene Demler ${ }^{1}$ \\ ${ }^{1}$ Department of Physics, Harvard University, Cambridge, Massachusetts 02138, USA \\ ${ }^{2}$ Department of Physics, Rice University, Houston, Texas 77005, USA
}

(Received 18 April 2006; published 29 November 2006)

\begin{abstract}
We consider spin-polarized mixtures of cold fermionic atoms on the BEC side of the Feshbach resonance. We demonstrate that a strongly anisotropic confining potential can give rise to a double-peak structure in the axial distribution of the density difference and a polarization-dependent aspect ratio of the minority species. Both phenomena appear as a result of the breakdown of the local density approximation for the phaseseparated regime. We speculate on the implications of our findings for the unitary regime.
\end{abstract}

DOI: 10.1103/PhysRevA.74.053626

PACS number(s): 03.75.Hh, 03.75.Ss

During the past few years, considerable experimental progress has been achieved in creating systems of strongly interacting ultracold fermions [1]. One of the primary motivations for this research effort is simulating strongly correlated electron systems such as high-temperature superconductors and quantum magnets. The number of particles used in experiments with cold atoms is large but not macroscopic, so they may also exhibit mesoscopic phenomena. In this paper, we discuss several important manifestations of such mesoscopic effects in spin-polarized mixtures of ultracold fermionic atoms in the vicinity of a Feshbach resonance [2-6]. Focusing on the BEC side of the resonance, we demonstrate that some of the unusual features observed in experiments by Partridge et al. [3], including a double-peak structure in the axial distribution of the density difference and a polarizationdependent aspect ratio of the minority species [7], appear as a result of strong anisotropy of a confining potential. Our starting point is a rigorous proof that neither of the two phenomena can take place when the confining potential is parabolic and the local density approximation (LDA) holds (this has also been noted in Ref. [6]). A recent preprint by Zwierlein and Ketterle [8] argued that the unharmonicity of the confining potential should have contributed to the doublepeak structure observed in Ref. [3]. In this paper, we show that even for a harmonic potential, but for a strongly anisotropic trap, such as the one used in experiments of Partridge et al., beyond-LDA corrections give rise to both the doublepeak structure in the axial density difference and a polarization-dependent aspect ratio.

In this paper, we consider spin-polarized fermion mixtures on the BEC side of the Feshbach resonance at $T=0$. At low temperatures and deep into the BEC limit, all minoritycomponent fermions are paired, forming stable bosonic molecules. So the system can be thought of as a Bose-Fermi mixture, where "bosons" are tightly bound molecules and "fermions" are excess or unpaired fermions of the majority species. Boson-boson (i.e., molecule-molecule) and bosonfermion (i.e., molecule-fermion) scattering lengths can be related to the scattering length between two species of fermions as [9-11]

$$
a_{\mathrm{bb}}=0.6 a, \quad a_{\mathrm{bf}}=1.18 a .
$$

Before presenting a formal analysis, we provide a simple qualitative picture of our results. The leading correction to the LDA comes from including gradient terms in expressions for the chemical potentials of bosons and fermions. The gradient term for bosons corresponds to the kinetic energy term of the Gross-Pitaevskii equation and has been thoroughly analyzed in the literature [14]. Gradient terms for fermions have also been considered in the context of nuclear physics (see Chap. 4 of Ref. [15]). Interestingly, it turns out that gradient terms for fermions have a small numerical prefactor, and have a negligible effect in the considered region of the parameters. For strongly anisotropic traps, gradient terms smooth the density distribution of bosons in the tightly confined radial direction at the boundary of the boson cloud (see the inset to Fig. 1). This means that there are "extra" bosons in the radial direction compared to the LDA model. These "extra" bosons interact repulsively with unpaired fermions and push them out in the axial direction toward the long tips of the trap, leading to a double-peak structure in the axial density of excess fermions (see Fig. 2). We point out that we

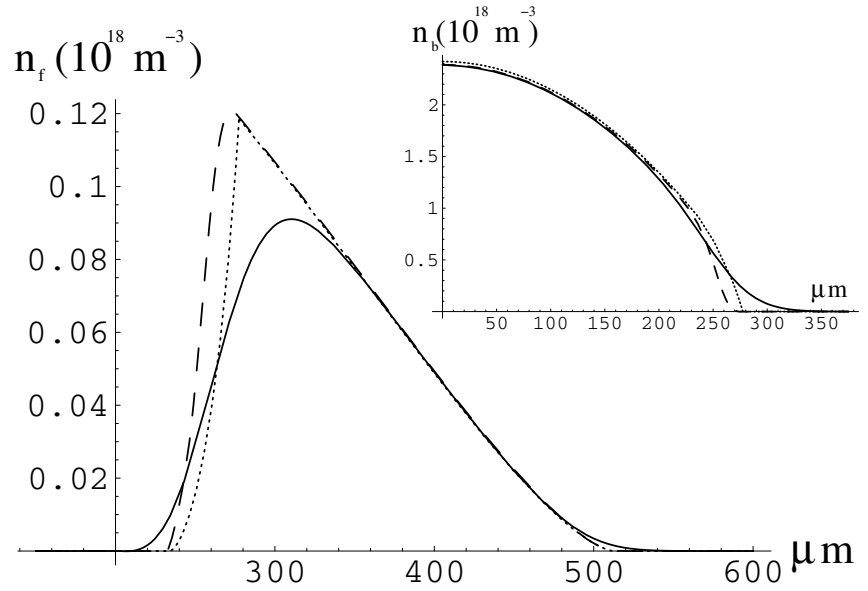

FIG. 1. Rescaled 3D densities of fermions in the Bose-Fermi model (2). These correspond to density differences of the majority and minority components $n_{f}=n_{\uparrow}-n_{\downarrow}$. LDA result (dotted), $n_{f}(r$ $=0, z=x$ ) (dashed), and $n_{f}(r=x / \Lambda, z=0)$ (solid) for harmonic confinement $\omega_{z} / 2 \pi=7.2 \mathrm{~Hz}$ and $\omega_{\perp} / 2 \pi=350 \mathrm{~Hz}$, scattering length corresponding to $B=754 \mathrm{G}, N_{\downarrow}+N_{\uparrow}=9.46 \times 10^{4}$, and $P=9.5 \%$. Inset: Bose densities for the same conditions. 


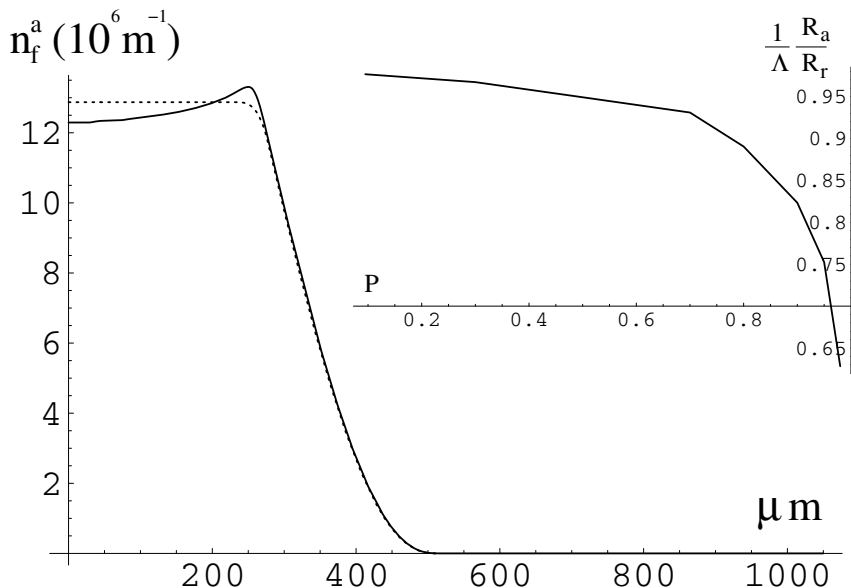

FIG. 2. Axial densities of fermions $\left(n_{f}^{a}=n_{\uparrow}^{a}-n_{\uparrow}^{a}\right)$ within LDA (dotted) and in the presence of beyond-LDA corrections (solid), for the same parameters as in Fig. 1. The inset shows the dependence of the anisotropy of the Bose cloud on polarization $P$, with other parameters being the same as in Fig. $1 . R_{a}$ and $R_{r}$ are obtained from the fits to the columnar density as discussed in the text.

do not find a critical value of polarization beyond which the double-peak structure appears, although the peak strength depends on polarization, scattering length, and the total number of particles in the system (see Fig. 3).

The evolution of the aspect ratio of the minority species is also easy to understand in the BEC limit that we consider. The aspect ratio of minority species is the aspect ratio of bosons (molecules). When the LDA applies, the aspect ratio of bosons is equal to the ratio of confining frequencies (in experiments reported in Ref. [3], this ratio is around 50). In the extreme limit of full polarization, one can consider a single boson (molecule) in an effective potential created by the confining potential and excess fermions. Solving a single-particle Schrödinger equation for the boson gives a wave function with the aspect ratio equal to the square root of the ratio of confining frequencies (this corresponds to the

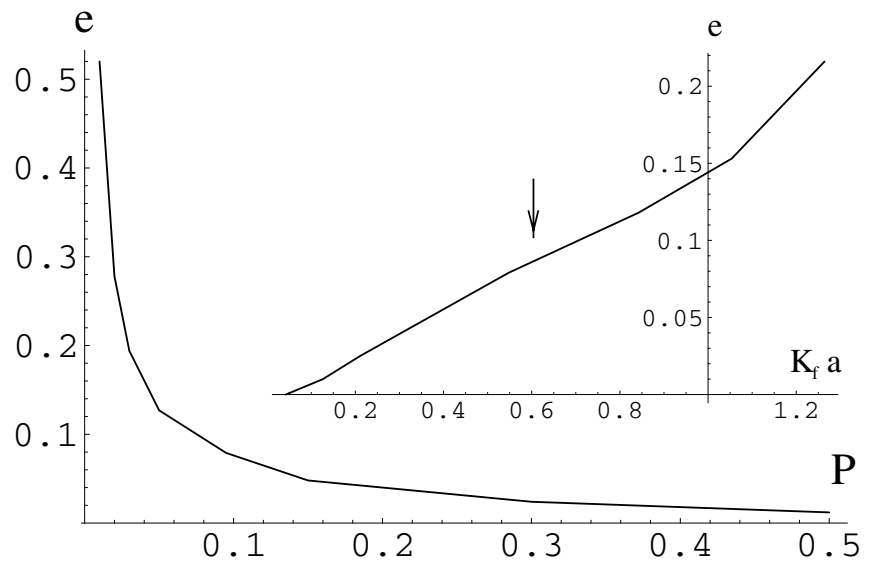

FIG. 3. Dependence of the strength of the double-peak structure $e$ on polarization $P$, with other parameters being the same as in Fig. 1. The inset shows how $e$ changes with $a$ when we move away from the point considered in Fig. 1 (marked with an arrow). $K_{f}$ is defined as in Ref. [3]. extreme breakdown of the LDA, see also [12]). When the number of bosons is small but finite, one does not find such a dramatic decrease of the aspect ratio, since even a small number of bosons leads to a change in the fermion distribution (see the inset to Fig. 2). However, this argument explains a general trend of decreasing aspect ratio of minority species with increasing polarization.

The microscopic model that we consider is the interacting Bose-Fermi Hamiltonian

$$
\begin{aligned}
H= & \int d^{3} r\left(\frac{\hbar^{2}}{2(2 m)} \nabla \Psi_{b}^{\dagger} \nabla \Psi_{b}+\frac{\hbar^{2}}{2 m} \nabla \Psi_{f}^{\dagger} \nabla \Psi_{f}\right. \\
& \left.+\frac{1}{2} g_{\mathrm{bb}} \Psi_{b}^{\dagger} \Psi_{b}^{\dagger} \Psi_{b} \Psi_{b}+g_{\mathrm{bf}} \Psi_{b}^{\dagger} \Psi_{f}^{\dagger} \Psi_{f} \Psi_{b}\right) .
\end{aligned}
$$

Here $\Psi_{f}\left(\Psi_{b}\right)$ is a fermion (boson) operator and $m$ is the original fermion mass. The interaction parameters are $g_{\mathrm{bb}}$ $=\frac{2 \pi \hbar^{2} a_{\mathrm{bb}}}{m}$ and $g_{\mathrm{bf}}=\frac{3 \pi \hbar^{2} a_{\mathrm{bf}}}{m}$. The Hamiltonian (2) with the parameters (1) can be rigorously derived in the dilute limit $n_{b}^{1 / 3} a \ll 1, n_{f}^{1 / 3} a \ll 1$, where $n_{b}$ and $n_{f}$ are local boson and fermion densities, from a single-channel model of a wide Feshbach resonance [11]. It has been found out numerically [16] in the absence of density imbalance that fermion mixture is well described by the model of interacting bosons up to $k_{f} a \leqq 1$.

Within the LDA and assuming a harmonic confinement, the density profiles are given as solutions of the following equations:

$$
\begin{gathered}
\mu_{f}\left(n_{b}, n_{f}\right)+V(x, y, z)=\text { const }_{1}, \\
\mu_{b}\left(n_{b}, n_{f}\right)+2 V(x, y, z)=\text { const }_{2},
\end{gathered}
$$

where $V(x, y, z)=\frac{m \omega_{z}^{2} z^{2}}{2}+\frac{m \omega_{\perp}^{2}\left(x^{2}+y^{2}\right)}{2}$. Notice that in the experiment of Ref. [3], the trap is highly anisotropic $\Lambda=\omega_{\perp} / \omega_{z}$ $=48.6 \gg 1$. Boson and fermion densities depend on coordinates $(x, y, z)$ through the potential $V(x, y, z)$ only. Hence we should have identical densities of each species for points that have the same value of the confining potential. Thus the aspect ratio of both clouds should always be equal to the trap anisotropy $\Lambda$. Then $3 \mathrm{D}$ densities along the $z$ axis, $n_{b}(z)$ $\equiv n_{b}(0,0, z)$ and $n_{f}(z) \equiv n_{f}(0,0, z)$, provide complete information about densities everywhere in the trap. From 3D densities $n_{b, f}(z)$ one can calculate axial densities as

$$
\begin{aligned}
n_{f}^{a}(z) & =\int d x d y n_{f}\left[\sqrt{\left(x^{2}+y^{2}\right) / \Lambda^{2}+z^{2}}\right] \\
& =\frac{2 \pi}{\Lambda^{2}} \int_{0}^{\infty} r d r n_{f}\left(\sqrt{r^{2}+z^{2}}\right)=\frac{\pi}{\Lambda^{2}} \int_{z^{2}}^{\infty} d t n_{f}(\sqrt{t}) .
\end{aligned}
$$

For $z>0$, the derivative of the axial density is

$$
\frac{d n_{f}^{a}(z)}{d z}=-\frac{2 \pi}{\Lambda^{2}} z n_{f}(z) \leqslant 0 .
$$

This provides a proof of the absence of peaks in the axial density away from $z=0$. We note that a similar statement can be made in the unitary and BCS regimes: if the density of the majority component is larger than that of the minority com- 
ponent at any point in the system, then the axial density cannot have a peak except at $z=0$. Within the LDA and harmonic trapping, phase separation is signaled not by the appearance of a double-peak structure in the unpaired fermion axial density, but by the existence of an extended region where the axial density difference has a zero derivative.

We now consider beyond-LDA corrections, which arise due to the spatial derivatives of the densities. At the meanfield level [13], correlations between the Bose and Fermi clouds are neglected and one treats the fermion (boson) density as providing an external potential for the bosons (fermions). Including the gradient corrections discussed earlier, up to second order in gradients of the densities, we find $[14,15]$

$$
\begin{aligned}
E= & \int d^{3} r\left[\frac{1}{2} g_{\mathrm{bb}} n_{b}^{2}+g_{\mathrm{bf}} n_{b} n_{f}+V(\mathbf{r})\left(n_{f}+2 n_{b}\right)\right. \\
& \left.+\frac{\hbar^{2}}{2 m}\left(\frac{\left|\nabla \sqrt{n_{b}}\right|^{2}}{2}+\frac{\left(6 \pi^{2} n_{f}\right)^{5 / 3}}{10 \pi^{2}}+\frac{\left(\nabla n_{f}\right)^{2}}{36 n_{f}}+\frac{\nabla^{2} n_{f}}{3}\right)\right] .
\end{aligned}
$$

In principle, for strong variations of the fermion density, higher-order terms in gradients have to be included. The gradient expansion above is not suitable for studies of shell structure [17], but since these effects are small in the case we are studying, this level of approximation is sufficient. Even though $p$-wave superfluidity of fermions due to bosoninduced interactions has been predicted (see, e.g., Ref. [5]), the value of the gap is exponentially small in $1 / n_{f}^{1 / 3} a$ and cannot affect significantly the density profiles obtained from the expression above.

Taking variations of $E$ with the density, one obtains corrections to the local chemical potential (valid in the region of nonzero fermion and boson densities),

$$
\begin{gathered}
\mu_{f}=\frac{\hbar^{2}}{2 m}\left[\left(6 \pi^{2} n_{f}\right)^{2 / 3}+\frac{1}{36}\left(\frac{\left(\nabla n_{f}\right)^{2}}{n_{f}^{2}}-\frac{\nabla^{2} n_{f}}{n_{f} / 2}\right)\right]+g_{\mathrm{bf}} n_{b}, \\
\mu_{b}=\frac{\hbar^{2}}{4 m} \frac{\nabla_{b}^{2} \sqrt{n_{b}}}{\sqrt{n_{b}}}+g_{\mathrm{bb}} n_{b}+g_{\mathrm{bf}} n_{f} .
\end{gathered}
$$

Notice that beyond-LDA corrections to the fermionic chemical potential carry a small prefactor of $1 / 36$ compared to the analogous term for bosons.

To investigate numerically density profiles in the presence of kinetic terms, we use the steepest descent method of functional minimization (used previously for bosons in anisotropic traps [18]). We investigate the effect of beyond-LDA corrections for typical parameters used in experiments (cf. Ref. [3]). In Fig. 1, we take harmonic confinement with $\omega_{z} / 2 \pi=7.2 \mathrm{~Hz}$ and $\omega_{\perp} / 2 \pi=350 \mathrm{~Hz}$, a scattering length that corresponds to $B=754 \mathrm{G}$, number of particles $N=N_{\downarrow}+N_{\uparrow}$ $=2 N_{b}+N_{f}=9.46 \times 10^{4}$, and polarization $P=\left(N_{\uparrow}-N_{\downarrow}\right) /\left(N_{\downarrow}\right.$ $\left.+N_{\uparrow}\right)=N_{f} /\left(N_{f}+2 N_{b}\right) \sim 10 \%$. The LDA density profile is almost completely phase separated due to the strong repulsion between bosons and fermions that spatially overlap only in a small region. In Fig. 1, we compare rescaled 3D density profiles in radial and axial directions with LDA results, and in Fig. 2 we do the same comparison for axial densities and identical values of the system parameters. Modification of
3D densities can be of the order of $30 \%$ for fermions, and is much stronger in the radial direction. This is expected, since the gradient corrections in the radial direction are $\Lambda^{2} \approx 2.4$ $\times 10^{3}$ times larger. We observe a peak in the axial density, with the density at the maximum about $10 \%$ higher than at $z=0$. The modification of the axial density is less prominent than changes in the 3D density profile due to the integration entering the definition of the axial density. Figure 3 shows the dependence of the relative strength of the double-peak structure $(e)$ on polarization $(P)$, where $e$ is defined as $e$ $=\left[\left.n_{f}^{a}(z)\right|_{\max }-n_{f}^{a}(0)\right] / n_{f}^{a}(0)$. It decreases with increasing $P$, since for a larger polarization a smaller fraction of the fermion cloud gets affected by the boundary of the boson cloud. Being essentially a finite-size effect, $e$ also decreases with increasing total number of particles. However, this dependence is pretty weak, since the size of the cloud depends weakly on the total number of atoms (only as $N^{1 / 6}$ for the unitary regime within the LDA). The inset to Fig. 3 shows the dependence of $e$ on scattering length $(a)$. As $a$ increases, the region of spatial overlap of the Bose and Fermi clouds shrinks due to stronger repulsion, and this leads to larger gradients and larger beyond-LDA corrections for the boson cloud. In addition, due to the enhanced repulsion, the fermions are more sensitive to the corrections to the boson cloud. Both of these effects lead to an increase of $e$ for larger $a$. We present results as a function of $K_{f} a$, where $K_{f}$ is defined as in Ref. [3]. Notice, however, that there is no "universality" in this curve: indeed, increasing the total number for fixed $a$ would increase $K_{f} a$, while $e$ would decrease, as discussed earlier. Although $K_{f} a$ in Fig. 3 is of the order of 1, the main physics we are interested in takes place at the boundary of the boson cloud, where local $k_{f} a$ is considerably smaller, so the treatment of the system as a Bose-Fermi mixture is well justified.

Beyond-LDA corrections increase the size of the boson cloud in the radial direction compared to the LDA result, thus reducing the aspect ratio of the bosonic cloud compared to $\Lambda$. As the polarization $(P)$ increases, for a fixed total number of atoms, the size of the bosonic cloud decreases and the importance of beyond-LDA effects grows. The inset to Fig. 2 shows the evolution of anisotropy as a function of polarization for harmonic confinement. $R_{a}$ and $R_{r}$ are defined as follows: (i) the columnar density $n_{b}^{c}(r, z)$ is obtained from $n_{b}(r, z)$, assuming radial symmetry in the $x-y$ plane; (ii) one then fits noninteracting-fermion density distributions to the columnar density along axial and radial directions, $n_{b}^{c}(r, 0)$ $=A\left(1-\frac{r^{2}}{R_{r}^{2}}\right)^{2}$ and $n_{b}^{c}(0, z)=B\left(1-\frac{z^{2}}{R_{a}^{2}}\right)^{2}$. Notice these functional forms are not always suitable and the fits provide just an estimate of the radii. The aspect ratio of the fermionic cloud does not get significantly modified in a harmonic confinement.

So far we discussed only the BEC regime, where controllable analytic theory is available. We developed a consistent qualitative picture of the appearance of peaks in the axial density difference, $n_{f}^{a}=n_{\uparrow}^{a}-n_{\downarrow}^{a}$, and a decrease of the aspect ratio of minority species with increasing polarization. Quantitatively, the numbers we obtain in the BEC regime are smaller than what is observed experimentally in Ref. [3] for the unitary regime, but of the same order of magnitude as the 
effects of unharmonicity discussed in Ref. [8]. Now we will comment on the relevance of our findings for the unitary limit, where most experiments are performed. Results from the BEC regime should not be directly extrapolated toward the unitary regime, since the treatment of the system as a Bose-Fermi mixture starts to break down, and the form of beyond-LDA corrections in the unitary regime is not known. Hence the statements made in this paragraph are only speculative. One can estimate, within the LDA, the chemical potential in the center of the trap based on Fig. 2C of Ref. [3]: for a typical configuration it is $\sim 10 \hbar \omega_{\perp}$, where $\hbar \omega_{\perp}$ is the radial level spacing. The chemical potential at the boundary of the inner cloud is about three to four times $\hbar \omega_{\perp}$, whereas the gradient contribution in the radial direction, which is neglected in the LDA, is $\sim \hbar^{2} \Lambda^{2} /\left[m(\Delta R)^{2}\right] \sim \hbar \omega_{\perp}$ (where $\Delta R$ is the difference in axial radii). It is thus clear that beyondLDA corrections might also be relevant for the unitary regime. The appearance of double peaks has been interpreted in Ref. [3] as evidence for phase separation of excess fermions from a paired central core. Although peaks should not appear in the LDA, the picture we have in the BEC limit provides some support to this interpretation. When excess fermions spatially overlap with the paired core, they are less sensitive to beyond-LDA corrections, which are important only at the boundary of the paired cloud. As phase separation takes place, excess fermions are expelled to the boundary, where beyond-LDA effects become more important. These corrections are stronger in the radial direction, hence fermions are pushed in the axial direction to larger $|z|$. This may give rise to a pronounced double-peak structure in the axial density. The high anisotropy of the trap is important for this scenario.

To summarize, we considered unbalanced fermions in the BEC limit for $T=0$. We proved a "theorem" that prohibits peaks in the axial density for $z \neq 0$ within the LDA and for the harmonic trapping potential. We showed that for strongly anisotropic confinement, beyond-LDA corrections produce a double-peak structure in the axial density, and change the aspect ratio of the inner cloud. We discussed the implications of our findings for the unitary regime.

We thank R. Hulet, M. Zwierlein, W. Ketterle, and H. Stoof for useful discussions. This work was partially supported by NSF Grant No. DMR-0132874, NSF Career Program, MIT-Harvard CUA and AFOSR.
[1] C. A. Regal, M. Greiner, and D. S. Jin, Phys. Rev. Lett. 92, 040403 (2004); M. Bartenstein et al., ibid. 92, 120401 (2004); M. W. Zwierlein et al., ibid. 92, 120403 (2004); J. Kinast et al., ibid. 92, 150402 (2004); T. Bourdel et al., ibid. 93, 050401 (2004); G. B. Partridge et al., ibid. 95, 020404 (2005); T. Stoferle et al., ibid. 96, 030401 (2006).

[2] M. W. Zwierlein, A. Schirotzek, C. H. Schunck, and W. Ketterle, Science 311, 492 (2006).

[3] G. B. Partridge, W. Li, R. I. Kamar, Y. Liao, and R. G. Hulet, Science 311, 503 (2006).

[4] P. F. Bedaque, H. Caldas, and G. Rupak, Phys. Rev. Lett. 91, 247002 (2003); A. Sedrakian, J. Mur-Petit, A. Polls, and H. Muther, Phys. Rev. A 72, 013613 (2005); C.-H. Pao and S.-T. Wu, S.-K. Yip, Phys. Rev. B 73, 132506 (2006); D. T. Son and M. A. Stephanov, Phys. Rev. A 74, 013614 (2006); D. E. Sheehy and L. Radzihovsky, Phys. Rev. Lett. 96, 060401 (2006); J. Dukelsky, G. Ortiz, S. M. A. Rombouts, and K. Van Houcke, ibid. 96, 180404 (2006); Kun Yang, e-print cond-mat/ 0508484; P. Pieri and G. C. Strinati, Phys. Rev. Lett. 96, 150404 (2006); J. Kinnunen, L. M. Jensen, and P. Torma, ibid. 96, 110403 (2006); W. Yi and L.-M. Duan, Phys. Rev. A 73, 031604(R) (2006); F. Chevy, Phys. Rev. Lett. 96, 130401 (2006); M. Haque and H. T. C. Stoof, Phys. Rev. A 74, 011602 (2006); H. Caldas, e-print cond-mat/0601148; Z.-C. Gu, G. Warner, and F. Zhou, cond-mat/0603091; X.-J. Liu and H. Hu, Europhys. Lett. 75, 364 (2006); H. Hu and X.-J. Liu, Phys. Rev. A 73, 051603(R) (2006); M. Iskin and C. A. R. Sa de Melo, e-print cond-mat/0604184; K. Machida, T. Mizushima, and M. Ichioka, Phys. Rev. Lett. 97, 120407 (2006).

[5] A. Bulgac, M. M. Forbes, and A. Schwenk, Phys. Rev. Lett. 97, 020402 (2006); D. V. Efremov and L. Viverit, Phys. Rev. B 65, 134519 (2002).

[6] T. N. De Silva and E. J. Mueller, Phys. Rev. A 73, 051602(R) (2006).

[7] R. Hulet, Presentation at APS March Meeting, Baltimore, 15 March 2006.

[8] M. W. Zwierlein and W. Ketterle, e-print cond-mat/0603489.

[9] G. V. Skorniakov and K. A. Ter-Martirosian, Sov. Phys. JETP 4, 648 (1957).

[10] D. S. Petrov, C. Salomon, and G. V. Shlyapnikov, Phys. Rev. Lett. 93, 090404 (2004).

[11] I. V. Brodsky et al., JETP Lett. 82, 273 (2005); J. Levinsen, and V. Gurarie, Phys. Rev. A 73, 053607 (2006).

[12] A. Gorlitz et al., Phys. Rev. Lett. 87, 130402 (2001).

[13] L. Viverit, C. J. Pethick, and H. Smith, Phys. Rev. A 61, 053605 (2000)

[14] L. Pitaevskii and S. Stringari, Bose-Einstein Condensation (Clarendon Press, Oxford, UK, 2003).

[15] M. Brack and R. K. Bhaduri, Semiclassical Physics, Frontiers in Physics Vol. 96 (Addison-Wesley, Reading, MA, 1997).

[16] G. E. Astrakharchik, J. Boronat, J. Casulleras, and S. Giorgini, Phys. Rev. Lett. 93, 200404 (2004).

[17] E. J. Mueller, Phys. Rev. Lett. 93, 190404 (2004).

[18] F. Dalfovo and S. Stringari, Phys. Rev. A 53, 2477 (1996); for each point we use about 1000 iterations for functions defined on a $100 \times 100$ grid. 